\title{
Revisit the Practice of Lymph Node Biopsy in Patients Diagnosed as Ductal Carcinoma In Situ Before Operation: A Retrospective Analysis of 682 Cases and Evaluate the Role of Breast MRI
}

\section{Hung-Wen Lai}

Changhua Christian Medical Foundation Changhua Christian Hospital

Yi Lin Chang ( $\square$ klaire_2014@outlook.com )

Changhua Christian Medical Foundation Changhua Christian Hospital https://orcid.org/0000-00018949-1034

\section{Shou-Tung Chen}

Changhua Christian Medical Foundation Changhua Christian Hospital

\section{Yu-Chun Chang}

Changhua Christian Medical Foundation Changhua Christian Hospital

\section{Wen-Pei Wu}

Changhua Christian Medical Foundation Changhua Christian Hospital

\section{Dar-Ren Chen}

Changhua Christian Medical Foundation Changhua Christian Hospital

Shou-Jen Kuo

Changhua Christian Medical Foundation Changhua Christian Hospital

\section{Research Article}

Keywords: ductal carcinoma in situ, upgrade, lymph node metastasis, sentinel lymph node biopsy (SLNB), mastectomy

Posted Date: February 23rd, 2021

DOl: https://doi.org/10.21203/rs.3.rs-237060/v1

License: (c) (i) This work is licensed under a Creative Commons Attribution 4.0 International License. Read Full License 


\section{Abstract}

Background: The optimal axillary lymph node (ALN) management strategy in patients diagnosed with ductal carcinoma in situ (DCIS) preoperatively remains controversial. The value of breast magnetic resonance imaging (MRI) to predict ALN metastasis pre-operative DCIS patients were evaluated.

Methods: Patients with primary DCIS with or without pre-operative breast MRI evaluation and underwent breast surgery were recruited from single institution. The value of breast MRI for ALN evaluation, predictors of breast \& ALN surgeries, upgrade from DCIS to invasive cancer, and ALN metastasis were analyzed.

Results: A total of 682 cases with pre-operative diagnosis of DCIS were enrolled in current study. The rate of upgrade to invasive cancer were found in $34.2 \%$ of specimen, and this upgrade rate is $23 \%$ for patients received breast conserving surgery and $40.7 \%$ for mastectomy $(p<0.01)$. Large pre-operative imaging tumor size and post-operative invasive component were risk factors to ALN metastasis. Breast MRI had $53.8 \%$ sensitivity, $77.8 \%$ specificity, $14.9 \%$ positive predictive value, $95.9 \%$ negative predictive value (NPV), and $76.2 \%$ accuracy to predict ALN metastasis in pre-OP DCIS patients. In MRI node negative breast cancer patients with MRI tumor size $<3 \mathrm{~cm}$, the NPV was $96.4 \%$, and all these false negative cases were N1. Pre-OP diagnosed DCIS patients with MRI tumor size $<3 \mathrm{~cm}$ and node negative suitable for BCS could safety omit SLNB if whole breast radiotherapy is to be performed.

Conclusion: Breast MRI had high NPV to predict ALN metastasis in pre-OP DCIS patients, which is useful and could be provided as shared decision-making reference.

\section{Introduction}

Lymph node evaluation plays important role of breast cancer staging and management, and had been evolved from axillary lymph node dissection (ALND) to sentinel lymph node biopsy (SLNB). In theory, ductal carcinoma in situ (DCIS) does not metastasize to adjacent lymph nodes, and axillary lymph node evaluation or surgery had limited role. DCIS as determined by pathologic analysis of biopsy specimens, however, does not preclude invasive disease in excised specimens, and up to $50 \%$ (range, $3.5-56 \%$ ) of core needle biopsy (CNB) or vacuum-assisted core biopsy (VACB) diagnosed DCIS would upgrade to have an invasive component (IC) [1-17]. Indication and adequacy of application of SLNB in lymph node evaluation of patients with pre-operative (pre-OP) DCIS diagnosed by biopsy remained a debated issue as SLNB remains an invasive procedure and not morbidity free[18-20].

In current practice guideline, SLNB was not routinely suggested for patients with pre-OP DCIS planned to receive breast conserving surgery (BCS) as the rate of upgrade to DCIS-IC is not so high in lesion suitable for local excision, and even if invasive component found a secondary SLNB could still be performed[21]. Furthermore, according to ACOSZ0011, even 1-2 positive SLNB could be managed with whole breast radiotherapy in T1-T2 tumor received BCS without ALND[22]. In patients with pre-OP DCIS and indicated for mastectomy, however, SLNB remained recommended as the upgrade rate is increased and there would 
be rare chance for secondary lymph node surgical biopsy. In recent studies, the rate of positive SLNB following mastectomy of patients with pre-OP biopsied DCIS was low (around 10\%-20\%), which raising the question of the need and value of routine SLNB in this particular group of patients in modern era of breast imaging[1, 23-28].

In our previous study we had showed pre-OP breast magnetic resonance image (MRI) had clinical benefit in predict DCIS with invasive component (DCIS-IC). Certain characteristics of breast MRI, like MRI evidence of NAC invasion, mass-like lesions, and measurable apparent diffusion coefficient area were significant predictors of DCIS-IC[29]. DCIS-IC was found to be the most important predictor of ALN metastasis in SLNB in patients with pre-OP DCIS. We hypothesized that pre-OP MRI could have potential role for ALN evaluation and prevent unnecessary SLNB in some conditions.

The aim of current study is to evaluate the role of ALN surgery in pre-OP biopsy diagnosed DCIS patients, and investigate the accuracy of breast MRI to predict ALN metastasis. The rate and predictors of upgrade from pre-OP DCIS to DCIS-IC, ALN metastasis, and potential of breast MRI to replace SLNB in pre-OP biopsy diagnosed DCIS patients would be analyzed and discussed.

\section{Materials And Methods}

\section{Patients}

Patients with primary DCIS as diagnosed by biopsy (mainly CNB, VACB or excision biopsy) with or without pre-OP breast MRI evaluation and underwent breast surgery during the period of January 2009 to December 2018 at the Changhua Christian Hospital (CCH), a tertiary medical center in central Taiwan, were retrospectively recruited from the breast cancer database. Patients without detailed clinicopathologic data were excluded (Fig. 1). The study was approved by the institutional review board (IRB) of CCH (CCH IRB No. 140404). The clinicopathologic factors gathered from the data base included age, tumor size, biopsy method, tumor grade, and status of estrogen receptor (ER), progesterone receptor (PR), and human epithelial growth factor receptor 2 (HER-2) expression.

\section{Definition The Adequacy Of Sentinel Lymph Node Biopsy (slnb):}

In patients whom SLNB was indicated, methylene blue and/or radioisotope (Tc99m) were used. Indication and threshold for surgical lymph biopsy in patients with pre-OP DCIS patients remained a controversial issue. When IC present, SLNB could be viewed as "adequate-treatment" while no axillary surgery seemed likely to "under-treatment". When pure DCIS found in permanent pathology, SLNB seemed "overtreatment" and patients without surgical lymph node biopsy viewed as "adequate-treatment". We apply the above mentioned: "adequate-treatment, under-treatment, over-treatment" to our patients according to their post-operative pathology and whether ALN surgery was performed. 
To evaluate the indication and adequacy of surgical lymph node biopsy of patients with pre-OP biopsy diagnosed DCIS patients, another group of pre-OP invasive cancer patients were identified for comparison. The risk of final ALN metastasis, the degree of ALN metastasis (N1 (1-3 nodes), N2(4-9 nodes), and N3(> 10 nodes) were compared between different groups of patients, namely pre-OP DCIS, post-OP pure DCIS, DCIS-IC, and invasive cancer patients.

\section{Predictors for upgrade from DCIS to DCIS with invasive component (DCIS-IC)}

Patients were separated into two groups, namely a DCIS group comprising patients with post-operative (post-OP) histopathologic evidence of pure DCIS and a DCIS-IC group comprising patients with post-OP evidence of DCIS with invasive component (i.e., basement membrane invasion, characterized immunohistochemically by the lack of p63 staining in myoepithelial cells).

\section{Magnetic Resonance Imaging (mri) And Protocol}

The sensitivity, specificity, positive predictive value (PPV), negative predictive value (NPV), accuracy of breast MRI in prediction of ALN metastasis was evaluated by comparing the concordance of preoperative breast MRI lymph node report and post-operative pathologic lymph node status. The protocol and method of breast MRI used in current study had been reported before[29], and a brief summary was described. MR imaging was performed with a Siemens MAGNETOM Verio3.0 Tesla MRI machine. All patients were imaged in the prone position with both breasts placed into a dedicated 16-channelbreast coil.

MR imaging protocols included the following: bilateral laxial turbo-spin-echo fat-suppressed T2-weighted imaging (TR/TE4630/70 ms; field of view $320 \mathrm{~mm}$; slice thickness $3 \mathrm{~mm}$; number of excitations 1), axial turbo-spin-echo T1 weighted imaging (TR/TE736/9.1 ms; field of view $320 \mathrm{~mm}$; slice thickness $3 \mathrm{~mm}$; number of excitations 1), and diffusion weighted imaging (DWI) (TR/TE5800/82 ms; field of view 360 $\mathrm{mm}$; slice thickness $3 \mathrm{~mm}$, with b values of 0,400 , and $800 \mathrm{sec} / \mathrm{mm} 2$ ). Dynamic contrast enhanced MR images (DCE-MRI) were obtained with a three-dimensional fat-suppressed volumetric interpolated breathhold examination (VIBE) sequence with parallel acquisition once before and five times after a bolus injection of gadobenate dimeglumine $(0.1 \mathrm{mmol} / \mathrm{kg})$. Both breasts were examined in the transverse plane at $60 \mathrm{~s}$ intervals in each phase of the dynamic studies. All of the MRI readings were interpreted by an experienced, board-certified breast imaging radiologist (HKW), who had a 35-years radiologist career and 15 years' experience of breast MRI.

\section{Statistical Analyses}

Data are expressed as mean \pm standard deviation for continuous variables. Categorical variables were compared using the chi-square test or Fisher's exact test when appropriate. The independent t-test was used to compare continuous variables. A P value of less than 0.05 was considered to indicate statistical 
significance; all tests were two-tailed. All statistical analyses were performed on a personal computer with the statistical package SPSS for Windows (Version19.0, SPSS, Chicago).

\section{Results}

According to inclusion and exclusion criteria, a total of 682 cases with pre-OP biopsy diagnosed DCIS were enrolled in current study. Among our patients, 252 (37\%) received BCS while 430 (63\%) received mastectomy, and surgical lymph node biopsy was performed in $90.9 \%$ of them. The clinicopathologic characteristics and management flow chart of pre-OP DCIS patients were shown in Table 1 \& Fig. 1. PostOP upgrade to invasive breast cancer were found in $34.2 \%$ (233/682) breast cancer specimens, and this upgrade rate is $23 \%(58 / 252)$ for patients received BCS and $40.7 \%(175 / 430)$ mastectomy (Table $2, p<$ $0.01)$. 
Table 1

Clinical presentations and demographic data of 682 patients with pre-operative diagnosis of ductal carcinoma in situ (DCIS)

\begin{tabular}{|c|c|}
\hline$N=682$ & Mean \pm SD (\%) \\
\hline Age, year & $52.3 \pm 10.1$ \\
\hline \multicolumn{2}{|l|}{ Location } \\
\hline Right & $328(48.1)$ \\
\hline Left & $354(51.9)$ \\
\hline \multicolumn{2}{|c|}{ Biopsy method(N/A = 3) } \\
\hline CNB & $421(62)$ \\
\hline Stereotactic biopsy & $167(24.6)$ \\
\hline Excisional biopsy & $88(12.9)$ \\
\hline MMG & $3(0.4)$ \\
\hline Tumor size, cm & $2.2 \pm 2.5$ \\
\hline \multicolumn{2}{|c|}{ Lymph node(N/A = 33) } \\
\hline Positive & $49(7.6)$ \\
\hline Negative & $600(92.4)$ \\
\hline \multicolumn{2}{|l|}{ Stage $(N / A=33)$} \\
\hline 0 & $390(60.1)$ \\
\hline I & 183(28.2) \\
\hline ॥ & $65(10.0)$ \\
\hline III & $11(1.7)$ \\
\hline \multicolumn{2}{|c|}{ Lymph node stage(N/A = 83) } \\
\hline No & $549(91.7)$ \\
\hline N1 & $40(6.7)$ \\
\hline N2 & $7(1.2)$ \\
\hline N3 & $3(0.5)$ \\
\hline Grade(N/A = 74) & \\
\hline
\end{tabular}

NA: not available, CNB: core needle biopsy, MMG: mammography guided biopsy, ER: estrogen receptor, PR: progesterone receptor, HER-2: human epithelial receptor type 2, OP: operation, DCIS: ductal carcinoma in situ, LCIS: lobular carcinoma in situ, ILC: infiltrating lobular carcinoma, IDC: infiltrating ductal carcinoma 


\begin{tabular}{|ll|}
\hline $\mathbf{N}=\mathbf{6 8 2}$ & Mean \pm SD (\%) \\
\hline I & $76(12.5)$ \\
\hline III & $337(55.4)$ \\
\hline ER(N/A = 24) & $195(32.1)$ \\
\hline Positive & $484(73.6)$ \\
\hline Negative & $174(26.4)$ \\
\hline PR(N/A = 33) & \\
\hline Positive & $438(67.5)$ \\
\hline Negative & $211(32.5)$ \\
\hline HER-2(N/A=203) & \\
\hline Positive & $178(37.2)$ \\
\hline Negative & $301(62.8)$ \\
\hline Post-OP Pathology & \\
\hline DCIS & $412(60.4)$ \\
\hline
\end{tabular}

NA: not available, CNB: core needle biopsy, MMG: mammography guided biopsy, ER: estrogen receptor, PR: progesterone receptor, HER-2: human epithelial receptor type 2, OP: operation, DCIS: ductal carcinoma in situ, LCIS: Iobular carcinoma in situ, ILC: infiltrating lobular carcinoma, IDC: infiltrating ductal carcinoma 


\begin{tabular}{|ll|}
\hline N $=682$ & Mean \pm SD (\%) \\
\hline LCIS & $5(0.7)$ \\
DCIS + LCIS & $7(1.0)$ \\
DCIS + microinvasive & $5(0.7)$ \\
& \\
& \\
& \\
DCIS + tubular carcinoma & \\
DCIS + mucinous carcinoma & $1(0.1)$ \\
IDC + DCIS & $3(0.4)$ \\
IDC & $228(33.4)$ \\
ILC & $9(1.3)$ \\
Other & $1(0.1)$ \\
\hline NA: not available, CNB: core needle biopsy, MMG: mammography guided biopsy, ER: estrogen \\
receptor, PR: progesterone receptor, HER-2: human epithelial receptor type 2, OP: operation, DCIS: \\
ductal carcinoma in situ, LCIS: lobular carcinoma in situ, ILC: infiltrating lobular carcinoma, IDC: \\
infiltrating ductal carcinoma
\end{tabular}


Table 2

Upgrade rate, adequacy of surgical lymph node biopsy, and rate of lymph node metastasis in patients diagnosed as ductal carcinoma in situ before operation.

\section{Upgrade ratio versus operation method}

\begin{tabular}{|c|c|c|c|c|}
\hline & BCS & Mastectomy & Total & $P$ value \\
\hline DCIS & 194 & 255 & $449(65.8 \%)$ & $<0.01$ \\
\hline DCIS-IC & 58 & 175 & $233(34.2 \%)$ & \\
\hline Total & $252(37 \%)$ & $430(63 \%)$ & 682 & \\
\hline \multicolumn{5}{|c|}{ Post-OP pathology and lymph node surgery } \\
\hline Post-OP pathology & DCIS & DCIS-IC & Total & $P$ value \\
\hline \multicolumn{5}{|l|}{ Axillary surgery } \\
\hline \multirow{2}{*}{$\begin{array}{l}\text { ALN } \\
\text { biopsy/dissection }\end{array}$} & $397(58.2 \%)$ & $223(32.7 \%)$ & 620 & 0.01 \\
\hline & over treatment & $\begin{array}{l}\text { adequate } \\
\text { treatment }\end{array}$ & & \\
\hline \multirow[t]{2}{*}{ No axillary surgery } & $52(7.6 \%)$ & $10(1.5 \%)$ & 62 & \\
\hline & $\begin{array}{l}\text { adequate } \\
\text { treatment }\end{array}$ & under-treatment & & \\
\hline Total & 449 & 233 & 682(100\%) & \\
\hline
\end{tabular}

\section{Pathology and lymph node metastasis distribution}

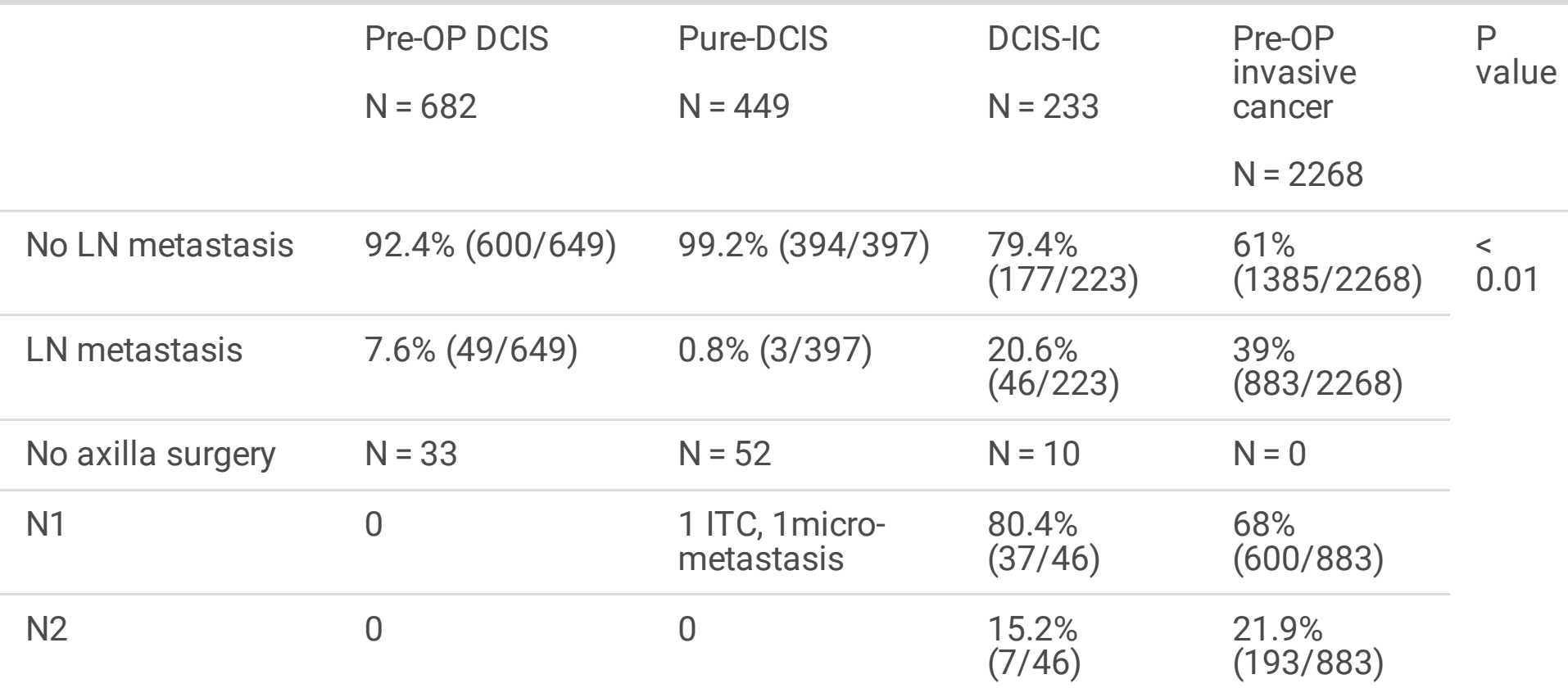

DCIS: ductal carcinoma in situ, DCIS-IC: ductal carcinoma in situ with invasive component, BCS: breast conserving surgery, ALN: axillary lymph node, OP: operation, LN: lymph node, ITC: isolated tumor cell 


\section{Upgrade ratio versus operation method}

\begin{tabular}{|c|c|c|c|c|}
\hline N3 & 0 & 1 & $4.3 \%(2 / 46)$ & $\begin{array}{l}10.2 \% \\
(90 / 883)\end{array}$ \\
\hline Total & & $419(100 \%)$ & $230(100 \%)$ & \\
\hline
\end{tabular}

Breast MRI lymph node metastasis evaluation

\begin{tabular}{|c|c|c|c|}
\hline & $\begin{array}{l}\text { MRI predict LN } \\
\text { metastasis }\end{array}$ & $\begin{array}{l}\text { MRI predict LN } \\
\text { negative }\end{array}$ & Total \\
\hline $\begin{array}{l}\text { Pathology LN } \\
\text { metastasis }\end{array}$ & 14 & 12 & 26 \\
\hline $\begin{array}{l}\text { Pathology LN } \\
\text { negative }\end{array}$ & 80 & 280 & 360 \\
\hline Total & 94 & 292 & 386 \\
\hline \multicolumn{4}{|c|}{ Sensitivity $=14 / 26=53.8 \%$} \\
\hline \multicolumn{4}{|c|}{ Specificity $=280 / 360=77.8 \%$} \\
\hline \multicolumn{4}{|c|}{ Positive predictive value (PPV) $=14 / 94=14.9 \%$} \\
\hline \multicolumn{4}{|c|}{ Negative predictive value (NPV) $=280 / 292=95.9 \%$} \\
\hline \multicolumn{4}{|c|}{ Accuracy $=(14+280) / 386=76.2 \%$} \\
\hline
\end{tabular}

The risk of ALN metastasis rate is overall $7.6 \%$ in pre-operative DCIS patients, $0.8 \%$ in post-operative pure DCIS, and $20.6 \%$ in post-operative DCIS-IC. The rate and distribution of ALN metastases of 682 patients with pre-OP diagnosed DCIS were compared with another cohort of 2268 pre-OP diagnosed invasive cancer patients and summarized in Table 2. According to post-OP pathology and whether SLNB was performed, $32.6 \%$ of SLNB was rated as "adequate-treatment", $58.4 \%$ "over-treatment", and $1.5 \%$ patients "under-treatment" while $7.6 \%$ patients with post-OP pure DCIS did not receive SLNB ("adequatemanagement", Fig. 1\& Table 2).

About 386 with detail pre-OP MRI ALN evaluation and post-OP pathologic report were analyzed for concordance, and MRI had $53.8 \%$ sensitivity, $77.8 \%$ specificity, $14.9 \%$ PPV, $95.9 \%$ NPV, and $76.2 \%$ overall accuracy (Table 2). In pre-OP MRI evaluated tumor size $<3 \mathrm{~cm}$ and no sign of ALN node metastasis breast cancer patients, the NPV was $96.4 \%$, and all the $3.6 \%(5 / 137)$ false negative (FN) cases were N1 patients. Among 70 patients received BCS, only 1 patient was found to have lymph node metastasis $(\mathrm{N} 1: 1 / 22)$. Another 67 patients received mastectomy, 4 patients were found to have lymph node metastasis (all N1: $2 / 13,1 / 5,1 / 2,1 / 3)$. 
The differences between patients who received breast (BCS versus mastectomy) and lymph node surgeries were compared and shown in Table 3. Larger pre-OP imaging tumor size is an independent risk factor for mastectomy and surgical ALN biopsy. The rate of surgical ALN biopsy rate is $96.3 \%(414 / 430)$ in mastectomy group, and $81.7 \%(206 / 252)$ in BCS (partial mastectomy) group $(P<0.01)$.OP method was also related to upgrade to DCIS, patients received total mastectomy had higher upgrade rate than partial mastectomy (BCS) patients $(40.7 \%$ versus $23 \%, P<0.01$, Table 3$)$. 
Table 3

Predictors of patients' selection for breast conserving surgery, mastectomy, and axillary lymph node surgery

\begin{tabular}{|c|c|c|c|c|c|c|}
\hline \multirow{3}{*}{ Pre-OP DCIS ( $N=682)$} & \multicolumn{3}{|c|}{$\begin{array}{l}\text { Total mastectomy versus partial } \\
\text { mastectomy (BCS) }\end{array}$} & \multicolumn{3}{|c|}{$\begin{array}{l}\text { Lymph node surgery versus no } \\
\text { surgery }\end{array}$} \\
\hline & $\begin{array}{l}\text { Total } \\
\text { mastectomy }\end{array}$ & $\begin{array}{l}\text { Partial } \\
\text { mastectomy }\end{array}$ & $\begin{array}{l}\mathrm{P} \\
\text { value }\end{array}$ & $\begin{array}{l}\text { No LN } \\
\text { surgery }\end{array}$ & $\begin{array}{l}\text { With LN } \\
\text { surgery }\end{array}$ & $\begin{array}{l}\mathrm{P} \\
\text { value }\end{array}$ \\
\hline & $N=430$ & $N=252$ & & $N=62$ & $N=620$ & \\
\hline Age, year & $51.9 \pm 9.9$ & $53.1 \pm 10.5$ & 0.14 & $54 \pm 12.2$ & $52.2 \pm 9.9$ & 0.17 \\
\hline Location & & & 0.33 & & & 0.31 \\
\hline Right & $213(49.5)$ & $115(45.6)$ & & $26(41.9)$ & $302(48.7)$ & \\
\hline Left & $217(50.5)$ & $137(54.4)$ & & $36(58.1)$ & $318(51.3)$ & \\
\hline Biopsy method & $N / A=3$ & $\mathrm{~N} / \mathrm{A}=0$ & $<.01$ & $\mathrm{~N} / \mathrm{A}=0$ & $N / A=3$ & $<.01$ \\
\hline CNB & 298(69.8) & $123(48.8)$ & & 19(30.6) & $402(65.2)$ & \\
\hline Stereotactic biopsy & 84(19.7) & $83(32.9)$ & & $23(37.1)$ & 144(23.3) & \\
\hline Excisional biopsy & $43(10.1)$ & $45(17.9)$ & & $18(29)$ & $70(11.3)$ & \\
\hline MMG & $2(0.5)$ & $1(0.4)$ & & $2(3.2)$ & $1(0.2)$ & \\
\hline $\begin{array}{l}\text { Sonogram Tumor size, } \\
\mathrm{cm}\end{array}$ & $2.2 \pm 1.2$ & $1.6 \pm 0.8$ & $\begin{array}{l}< \\
0.01\end{array}$ & $1.7 \pm 1.4$ & $2 \pm 1.1$ & 0.11 \\
\hline $\begin{array}{l}\text { Mammogram Tumor } \\
\text { size, cm }\end{array}$ & $2.9 \pm 1.3$ & $2.0 \pm 0.9$ & $\begin{array}{l}< \\
0.01\end{array}$ & $1.6 \pm 0.4$ & $2.6 \pm 1.2$ & 0.10 \\
\hline MRI Tumor size, $\mathrm{cm}$ & $4.5 \pm 2.3$ & $2.8 \pm 1.4$ & $<.01$ & $2.7 \pm 1.7$ & $4 \pm 2.2$ & $<.01$ \\
\hline $\begin{array}{l}\text { Pathology Tumor size } \\
\text { cm (invasive) }\end{array}$ & $2.6 \pm 2.9$ & $1.7 \pm 1.6$ & $<.01$ & $1.8 \pm 1.7$ & $2.3 \pm 2.6$ & 0.22 \\
\hline $\begin{array}{l}\text { Gross Tumor size, } \mathrm{cm}^{\#} \\
\text { (invasive + non-invasive) }\end{array}$ & $4 \pm 2.6$ & $2.2 \pm 1.4$ & $\begin{array}{l}< \\
0.01\end{array}$ & $2.6 \pm 1.8$ & $3.5 \pm 2.4$ & 0.04 \\
\hline Stage & $N / A=11$ & $\mathrm{~N} / \mathrm{A}=22$ & $<.01$ & $\mathrm{~N} / \mathrm{A}=12$ & $\mathrm{~N} / \mathrm{A}=21$ & 0.03 \\
\hline
\end{tabular}

*Pathology Tumor size: invasive component tumor size, " Gross Tumor size: invasive + non-invasive component tumor size,

MMG: mammography guided biopsy, LN: lymph node, SLNB: sentinel lymph node biopsy, ALND: axillary lymph node dissection, ND: not done, OP: operation, DCIS: ductal carcinoma in situ, TNBC: triple negative breast cancer, ER: estrogen receptor, PR: progesterone receptor, HER-2: human epithelial grow receptor type 2, N/A: not available 


\begin{tabular}{|c|c|c|c|c|c|c|}
\hline \multirow[b]{2}{*}{0} & \multicolumn{3}{|c|}{$\begin{array}{l}\text { Total mastectomy versus partial } \\
\text { mastectomy (BCS) }\end{array}$} & \multicolumn{3}{|c|}{$\begin{array}{l}\text { Lymph node surgery versus no } \\
\text { surgery }\end{array}$} \\
\hline & $226(53.9)$ & 164(71.3) & & $40(80)$ & $350(58.4)$ & \\
\hline I & $130(31)$ & $53(23)$ & & $8(16)$ & $175(29.2)$ & \\
\hline II & $54(12.9)$ & $11(4.8)$ & & $2(4)$ & $63(10.5)$ & \\
\hline III & $9(2.1)$ & $2(0.9)$ & & $0(0)$ & $11(1.8)$ & \\
\hline Grade & $N / A=38$ & $N / A=36$ & $\begin{array}{l}< \\
0.01\end{array}$ & $\mathrm{~N} / \mathrm{A}=19$ & $\mathrm{~N} / \mathrm{A}=55$ & 0.14 \\
\hline I & $39(9.9)$ & $37(17.1)$ & & $6(14.0)$ & $70(12.4)$ & \\
\hline II & $215(54.8)$ & $122(56.5)$ & & $29(67.4)$ & $308(54.5)$ & \\
\hline III & 138(35.2) & $57(26.4)$ & & $8(18.6)$ & 187(33.1) & \\
\hline ER & $N / A=9$ & $\mathrm{~N} / \mathrm{A}=15$ & $\begin{array}{l}< \\
0.01\end{array}$ & $N / A=11$ & $\mathrm{~N} / \mathrm{A}=13$ & 0.25 \\
\hline Positive & 293(69.6) & 191(80.6) & & 41(80.4) & $443(73.0)$ & \\
\hline Negative & $128(30.4)$ & 46(19.4) & & 10(19.6) & 164(27.0) & \\
\hline PR & $\mathrm{N} / \mathrm{A}=13$ & $N / A=20$ & $\begin{array}{l}< \\
0.01\end{array}$ & $\mathrm{~N} / \mathrm{A}=11$ & $N / A=22$ & 0.15 \\
\hline Positive & $259(62.1)$ & 179(77.2) & & $39(76.5)$ & $399(66.7)$ & \\
\hline Negative & 158(37.9) & $53(22.8)$ & & $12(23.5)$ & 199(33.3) & \\
\hline HER-2(N/A = 203) & $\mathrm{N} / \mathrm{A}=121$ & $N / A=82$ & 0.53 & $N / A=29$ & $\mathrm{~N} / \mathrm{A}=174$ & 0.64 \\
\hline Positive & 118(38.2) & $60(35.3)$ & & 11(33.3) & 167(37.4) & \\
\hline Negative & 191(61.8) & $110(64.7)$ & & $22(66.7)$ & $279(62.6)$ & \\
\hline Subtype & $N / A=68$ & $\mathrm{~N} / \mathrm{A}=52$ & $\begin{array}{l}< \\
0.01\end{array}$ & $N / A=24$ & $\mathrm{~N} / \mathrm{A}=96$ & 0.17 \\
\hline Luminal A & 198(54.7) & $133(66.5)$ & & 27(71.1) & $304(58.0)$ & \\
\hline Luminal B1 & $23(6.4)$ & $11(5.5)$ & & $2(5.3)$ & $32(6.1)$ & \\
\hline Luminal B2 & $50(13.8)$ & $32(16.0)$ & & 7(18.4) & $75(14.3)$ & \\
\hline
\end{tabular}

*Pathology Tumor size: invasive component tumor size, "Gross Tumor size: invasive + non-invasive component tumor size,

MMG: mammography guided biopsy, LN: lymph node, SLNB: sentinel lymph node biopsy, ALND: axillary lymph node dissection, ND: not done, OP: operation, DCIS: ductal carcinoma in situ, TNBC: triple negative breast cancer, ER: estrogen receptor, PR: progesterone receptor, HER-2: human epithelial grow receptor type 2, N/A: not available 


\begin{tabular}{|c|c|c|c|c|c|c|}
\hline \multirow[b]{2}{*}{ HER-2(+) } & \multicolumn{3}{|c|}{$\begin{array}{l}\text { Total mastectomy versus partial } \\
\text { mastectomy (BCS) }\end{array}$} & \multicolumn{3}{|c|}{$\begin{array}{l}\text { Lymph node surgery versus no } \\
\text { surgery }\end{array}$} \\
\hline & $56(15.5)$ & $15(7.5)$ & & $2(5.3)$ & 69(13.2) & \\
\hline TNBC & $35(9.7)$ & $9(4.5)$ & & $0(0)$ & $44(8.4)$ & \\
\hline Post-OP pathology & & & $<.01$ & & & $<.01$ \\
\hline DCIS & $255(59.3)$ & 194(77) & & $52(83.9)$ & $397(64)$ & \\
\hline Upgrade & $175(40.7)$ & $58(23)$ & & 10(16.1) & 223(36) & \\
\hline Ki-67 & $N / A=313$ & $N / A=221$ & $<.01$ & $\mathrm{~N} / \mathrm{A}=57$ & $N / A=477$ & 0.92 \\
\hline$\leqq 20$ & $67(57.3)$ & $25(80.6)$ & & $3(60)$ & $89(62.2)$ & \\
\hline$>20$ & $50(42.7)$ & $6(19.4))$ & & $2(40)$ & $54(37.8)$ & \\
\hline MRI LN metastasis & $N A=166$ & $\mathrm{~N} / \mathrm{A}=109$ & $\begin{array}{l}<.05 \\
0.05\end{array}$ & $N A=28$ & $N / A=247$ & 0.10 \\
\hline Yes & $69(26.1)$ & $25(17.5)$ & & $30(88.2)$ & $90(24.1)$ & \\
\hline No & 195(73.9) & $118(82.5)$ & & $4(11.8)$ & $283(75.9)$ & \\
\hline Lymph node stage & $N / A=60$ & $N / A=23$ & $\begin{array}{l}< \\
0.01\end{array}$ & $\mathrm{~N} / \mathrm{A}=15$ & $N / A=68$ & 0.20 \\
\hline NO & $327(88.4)$ & 222(96.9) & & $47(100)$ & $502(90.9)$ & \\
\hline N1 & $35(9.5)$ & $5(2.2))$ & & $0(0)$ & $40(7.2)$ & \\
\hline N2 & $6(1.6)$ & $1(0.4)$ & & $0(0)$ & $7(1.3)$ & \\
\hline N3 & $2(0.5)$ & $1(0.4)$ & & $0(0)$ & $3(0.5)$ & \\
\hline LN Metastasis & $N / A=8$ & $N / A=25$ & $<.01$ & $N / A=29$ & $\mathrm{~N} / \mathrm{A}=4$ & 0.09 \\
\hline Yes & $42(10)$ & $7(3.1)$ & & $0(0)$ & $49(8)$ & \\
\hline No & $380(90)$ & $220(96.9)$ & & $33(100)$ & $567(92)$ & \\
\hline OP method & - & - & - & & & $\begin{array}{l}< \\
0.01\end{array}$ \\
\hline
\end{tabular}

*Pathology Tumor size: invasive component tumor size, ${ }^{\#}$ Gross Tumor size: invasive + non-invasive component tumor size,

MMG: mammography guided biopsy, LN: lymph node, SLNB: sentinel lymph node biopsy, ALND: axillary lymph node dissection, ND: not done, OP: operation, DCIS: ductal carcinoma in situ, TNBC: triple negative breast cancer, ER: estrogen receptor, PR: progesterone receptor, HER-2: human epithelial grow receptor type 2, N/A: not available 


\begin{tabular}{|c|c|c|c|c|c|}
\hline \multirow[b]{2}{*}{ Total } & \multicolumn{3}{|c|}{$\begin{array}{l}\text { Total mastectomy versus partial } \\
\text { mastectomy (BCS) }\end{array}$} & \multicolumn{2}{|c|}{$\begin{array}{l}\text { Lymph node surgery versus no } \\
\text { surgery }\end{array}$} \\
\hline & - & - & - & $16(25.8)$ & $414(66.8)$ \\
\hline Partial & - & - & - & $46(74.2)$ & 206(33.2) \\
\hline Method LN & & & $\begin{array}{l}< \\
0.01\end{array}$ & - & - \\
\hline SLNB & $350(81.4)$ & 178(70.6) & & - & - \\
\hline SLNB + ALND & $31(7.2)$ & $18(7.1)$ & & - & - \\
\hline ALND & $33(7.7)$ & $10(4)$ & & - & - \\
\hline ND & 16(3.7) & $46(18.3)$ & & - & - \\
\hline \multicolumn{6}{|c|}{$\begin{array}{l}\text { *Pathology Tumor size: invasive component tumor size, }{ }^{\#} \text { Gross Tumor size: invasive + non-invasive } \\
\text { component tumor size, }\end{array}$} \\
\hline \multicolumn{6}{|c|}{$\begin{array}{l}\text { MMG: mammography guided biopsy, LN: lymph node, SLNB: sentinel lymph node biopsy, ALND: } \\
\text { axillary lymph node dissection, ND: not done, OP: operation, DCIS: ductal carcinoma in situ, TNBC: } \\
\text { triple negative breast cancer, ER: estrogen receptor, PR: progesterone receptor, HER-2: human epithelial } \\
\text { grow receptor type 2, N/A: not available }\end{array}$} \\
\hline
\end{tabular}

Predictors for post-OP upgraded to DCIS-IC were shown in Table 4, and larger tumor size, ER and/or PR negative tumors were associated with upgrade to DCIS-IC. Predictors for LN metastasis for patients with pre-OP diagnosed DCIS were evaluated, pre-OP imaging tumor size, DCIS-IC, and MRI predicted lymph node metastasis were risk factors to lymph node metastasis. In multivariate analysis, imaging tumor size (Odds ratio, OR $=1.93)$, DCIS-IC $(\mathrm{OR}=34.9)$ remained important factors (Table 5). 
Table 4

Predictors for upgrade to ductal carcinoma in situ (DCIS) with invasive component (IC) in pre-operative biopsy diagnosed DCIS patients

\begin{tabular}{|c|c|c|c|}
\hline$N=682$ & $\begin{array}{l}\text { Post-OP pure DCIS ( } \mathrm{N}= \\
\text { 449) }\end{array}$ & $\begin{array}{l}\text { Post-OP upgrade to DCIS-IC ( } N= \\
\text { 233) }\end{array}$ & $\begin{array}{l}P \\
\text { value }\end{array}$ \\
\hline Age, year & $52 \pm 9.8$ & $52.9 \pm 10.8$ & 0.29 \\
\hline Location & & & 0.62 \\
\hline Right & $219(48.8)$ & $109(46.8)$ & \\
\hline Left & $230(51.2)$ & $124(53.2)$ & \\
\hline Biopsy method & $\mathrm{N} / \mathrm{A}=2$ & $N / A=1$ & $<0.01$ \\
\hline CNB & $233(52.1)$ & 188(81) & \\
\hline Stereotactic biopsy & $140(31.3)$ & $27(11.6)$ & \\
\hline Excisional biopsy & 71(15.9) & $17(7.3)$ & \\
\hline MMG & $3(0.7)$ & $0(0)$ & \\
\hline Sonogram Tumor size, $\mathrm{cm}$ & $1.9 \pm 1.1$ & $2.2 \pm 1.2$ & $<0.01$ \\
\hline $\begin{array}{l}\text { Mammogram Tumor size, } \\
\mathrm{cm}\end{array}$ & $2.4 \pm 1.3$ & $2.8 \pm 1.1$ & 0.14 \\
\hline MRI Tumor size, $\mathrm{cm}$ & $3.6 \pm 2.2$ & $4.5 \pm 2.1$ & $<0.01$ \\
\hline $\begin{array}{l}\text { Pathology Tumor size } \\
\mathrm{cm}\end{array}$ & $2.7 \pm 2.7$ & $1.4 \pm 1.9$ & $<0.01$ \\
\hline Gross Tumor size ${ }^{\#}, \mathrm{~cm}$ & $3.3 \pm 2.4$ & $3.8 \pm 2.4$ & 0.02 \\
\hline Stage $(\mathrm{N} / \mathrm{A}=33)$ & $\mathrm{N} / \mathrm{A}=31$ & $N / A=2$ & $<0.01$ \\
\hline 0 & $381(91.1)$ & $9(3.9)$ & \\
\hline 1 & $26(6.2)$ & $157(68.0)$ & \\
\hline ॥ & $10(2.4)$ & $55(23.8)$ & \\
\hline III & $1(0.2)$ & $10(4.3)$ & \\
\hline Grade $(\mathrm{N} / \mathrm{A}=74)$ & $\mathrm{N} / \mathrm{A}=44$ & $N / A=30$ & $<0.01$ \\
\hline
\end{tabular}

*Pathology Tumor size: invasive component tumor size, ${ }^{*}$ Gross Tumor size: invasive + non-invasive component tumor size,

MMG: mammography guided biopsy, LN: lymph node, SLNB: sentinel lymph node biopsy, ALND: axillary lymph node dissection, ND: not done, OP: operation, DCIS: ductal carcinoma in situ, TNBC: triple negative breast cancer, ER: estrogen receptor, PR: progesterone receptor, HER-2: human epithelial grow receptor type 2, N/A: not available 


\begin{tabular}{|c|c|c|c|}
\hline$N=682$ & $\begin{array}{l}\text { Post-OP pure DCIS (N = } \\
449)\end{array}$ & $\begin{array}{l}\text { Post-OP upgrade to DCIS-IC (N = } \\
\text { 233) }\end{array}$ & $\begin{array}{l}P \\
\text { value }\end{array}$ \\
\hline I & $28(6.9)$ & $48(23.6)$ & \\
\hline II & 211(52.1) & $126(62.1)$ & \\
\hline III & 166(41.0) & $29(14.3)$ & \\
\hline ER & $N / A=24$ & & $<0.01$ \\
\hline Positive & $335(78.8)$ & 149(63.9) & \\
\hline Negative & $90(21.2)$ & $84(36.1)$ & \\
\hline$P R(N / A=33)$ & $N / A=33$ & & $<0.01$ \\
\hline Positive & $303(72.8)$ & 135(57.9) & \\
\hline Negative & $113(27.2)$ & $98(42.1)$ & \\
\hline HER-2 (N/A = 203) & $\mathrm{N} / \mathrm{A}=199$ & $\mathrm{~N} / \mathrm{A}=4$ & 0.25 \\
\hline Positive & $99(39.6)$ & $79(34.5)$ & \\
\hline Negative & 151(60.4) & 150(65.5) & \\
\hline MRI LN metastasis & $\mathrm{N} / \mathrm{A}=184$ & $\mathrm{~N} / \mathrm{A}=91$ & 0.01 \\
\hline Yes & $50(18.9)$ & $44(31.0)$ & \\
\hline No & 215(81.1) & $98(69.0)$ & \\
\hline Lymph node stage & $\mathrm{N} / \mathrm{A}=61$ & $N / A=22$ & $<0.01$ \\
\hline NO & 385(99.2) & 164(77.7) & \\
\hline N1 & $2(0.5)$ & 38(18.0) & \\
\hline N2 & $0(0)$ & $7(3.3)$ & \\
\hline N3 & $1(0.3)$ & $2(0.9)$ & \\
\hline OP method & & & $<0.01$ \\
\hline Total & 255(56.8) & 175(75.1) & \\
\hline Partial & 194(43.2) & $58(24.9)$ & \\
\hline
\end{tabular}

*Pathology Tumor size: invasive component tumor size, ${ }^{\#}$ Gross Tumor size: invasive + non-invasive component tumor size,

MMG: mammography guided biopsy, LN: lymph node, SLNB: sentinel lymph node biopsy, ALND: axillary lymph node dissection, ND: not done, OP: operation, DCIS: ductal carcinoma in situ, TNBC: triple negative breast cancer, ER: estrogen receptor, PR: progesterone receptor, HER-2: human epithelial grow receptor type 2, N/A: not available 


\begin{tabular}{|c|c|c|c|}
\hline$N=682$ & $\begin{array}{l}\text { Post-OP pure DCIS ( } \mathrm{N}= \\
\text { 449) }\end{array}$ & $\begin{array}{l}\text { Post-OP upgrade to DCIS-IC ( } \mathrm{N}= \\
\text { 233) }\end{array}$ & $\begin{array}{l}P \\
\text { value }\end{array}$ \\
\hline LN Meta & $N / A=30$ & $N / A=3$ & $<0.01$ \\
\hline Yes & $3(0.7)$ & $46(20.0)$ & \\
\hline No & 416(99.3) & 184(80.0) & \\
\hline Method_LN & & & $<0.01$ \\
\hline SLNB & $365(81.3)$ & 163(70.0) & \\
\hline SLNB + ALND & $14(3.1)$ & $35(15.0)$ & \\
\hline ALND & $18(4.0)$ & $25(10.7)$ & \\
\hline ND & $52(11.6)$ & $10(4.3)$ & \\
\hline \multicolumn{4}{|c|}{$\begin{array}{l}\text { *Pathology Tumor size: invasive component tumor size, }{ }^{\#} \text { Gross Tumor size: invasive }+ \text { non-invasive } \\
\text { component tumor size, }\end{array}$} \\
\hline \multicolumn{4}{|c|}{$\begin{array}{l}\text { MMG: mammography guided biopsy, LN: lymph node, SLNB: sentinel lymph node biopsy, ALND: } \\
\text { axillary lymph node dissection, ND: not done, OP: operation, DCIS: ductal carcinoma in situ, TNBC: } \\
\text { triple negative breast cancer, ER: estrogen receptor, PR: progesterone receptor, HER-2: human epithelial } \\
\text { grow receptor type 2, N/A: not available }\end{array}$} \\
\hline
\end{tabular}


Table 5

Univariate and multivariate for factors related to lymph node metastasis in patients with pre-operative biopsy diagnosed ductal carcinoma in situ

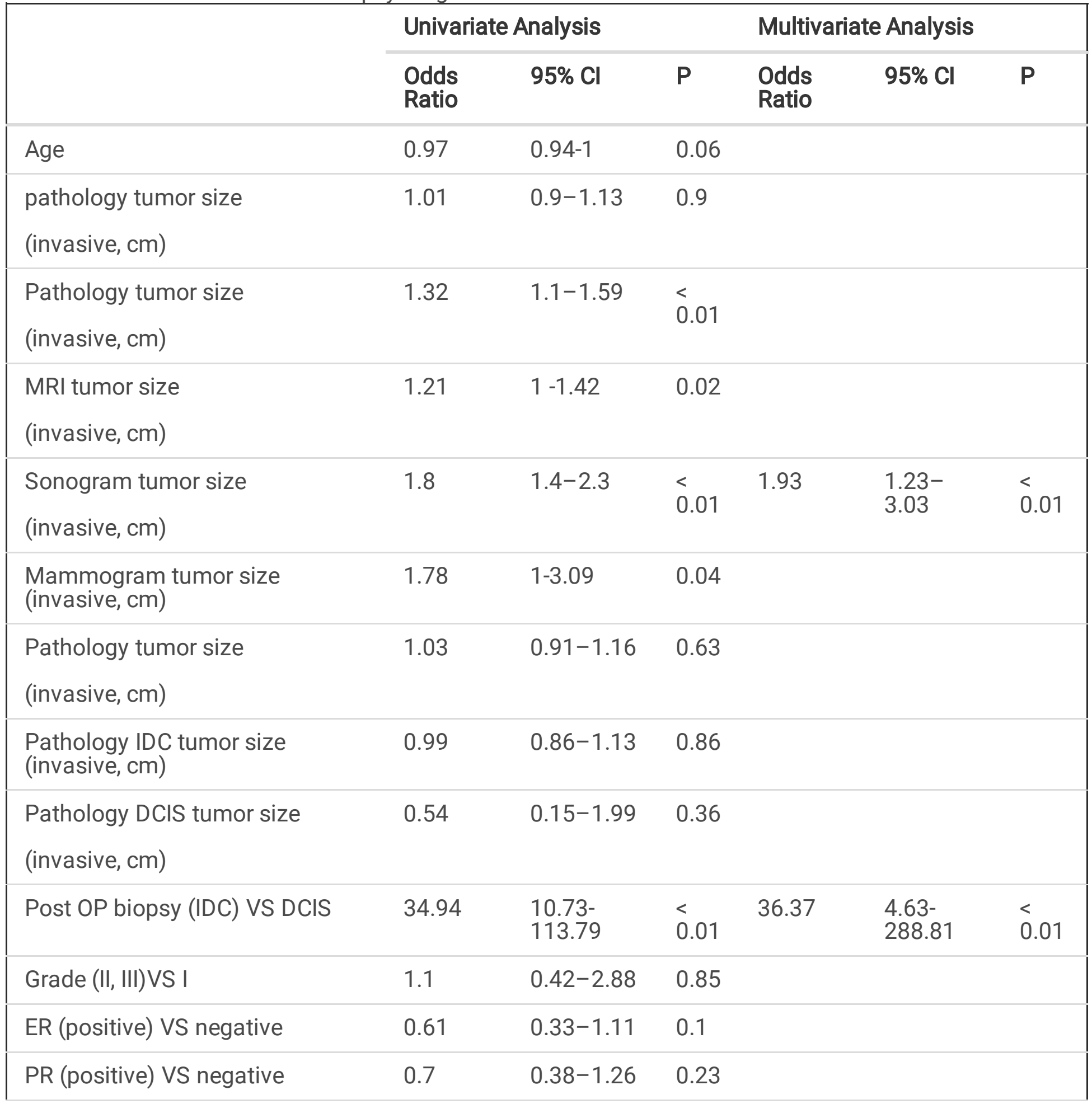

*Pathology Tumor size: invasive component tumor size, ${ }^{\# G r o s s ~ T u m o r ~ s i z e: ~ i n v a s i v e ~+~ n o n-i n v a s i v e ~}$ component tumor size,

MMG: mammography guided biopsy, LN: lymph node, SLNB: sentinel lymph node biopsy, ALND: axillary lymph node dissection, ND: not done, OP: operation, DCIS: ductal carcinoma in situ, TNBC: triple negative breast cancer, ER: estrogen receptor, PR: progesterone receptor, HER-2: human epithelial grow receptor type 2, N/A: not available 


\begin{tabular}{|c|c|c|c|c|c|c|}
\hline & \multicolumn{3}{|c|}{ Univariate Analysis } & \multicolumn{3}{|c|}{ Multivariate Analysis } \\
\hline & $\begin{array}{l}\text { Odds } \\
\text { Ratio }\end{array}$ & $95 \% \mathrm{Cl}$ & $\mathbf{P}$ & $\begin{array}{l}\text { Odds } \\
\text { Ratio }\end{array}$ & $95 \% \mathrm{Cl}$ & $\mathbf{P}$ \\
\hline HER-2 (positive) VS negative & 0.89 & $0.47-1.68$ & 0.72 & & & \\
\hline $\mathrm{Ki}-67(>20) \mathrm{VS} \leqq 20$ & 1.64 & $0.71-3.81$ & 0.25 & & & \\
\hline $\begin{array}{l}\text { MRI lymph node metastasis } \\
\text { (Y)VS N }\end{array}$ & 4.08 & $1.82-9.18$ & $\begin{array}{l}< \\
0.01\end{array}$ & 2.2 & $\begin{array}{l}0.83- \\
5.83\end{array}$ & 0.11 \\
\hline \multicolumn{7}{|c|}{$\begin{array}{l}\text { *Pathology Tumor size: invasive component tumor size, }{ }^{\#} \text { Gross Tumor size: invasive + non-invasive } \\
\text { component tumor size, }\end{array}$} \\
\hline \multicolumn{7}{|c|}{$\begin{array}{l}\text { MMG: mammography guided biopsy, LN: lymph node, SLNB: sentinel lymph node biopsy, ALND: } \\
\text { axillary lymph node dissection, ND: not done, OP: operation, DCIS: ductal carcinoma in situ, TNBC: } \\
\text { triple negative breast cancer, ER: estrogen receptor, PR: progesterone receptor, HER-2: human epithelia } \\
\text { grow receptor type 2, N/A: not available }\end{array}$} \\
\hline
\end{tabular}

\section{Discussion}

In the current study, we enrolled 682 pre-OP DCIS patients and compared ALN metastasis pattern with another cohort of 2268 pre-OP diagnosed invasive cancer. We found $34.2 \%$ of these pre-OP DCIS patients upgraded to DCIS-IC in final pathology, and this upgrade rate was consistent with literate reported range (3.5-56\%)[1-17]. The risk of ALN metastasis rate varied widely depends on the pre-operative pathology, and in our current study, the ALN metastasis rate is $7.6 \%$ in pre-OP DCIS patients, and up to $39 \%$ in pre-OP invasive cancer group (Table 2). In patients with post-OP pure DCIS, the LN metastasis rate is lowest (0.8\%), and ALN metastasis was found in $20.6 \%$ of patients with DCIS-IC. We also demonstrated that the ALN disease burden (N1-3) varied among different categories of patients (Table 2). These data reminded us the differences of ALN metastatic risk in each category of patients, and more tailored or individualized ALN evaluation strategy should be offered.

Indication and threshold for surgical lymph biopsy in patients with pre-OP biopsy diagnosed DCIS remained a controversy issue for decades. When invasive component present, SLNB was viewed as "adequate-treatment" while no axillary surgery seemed likely to "under-treatment". When pure DCIS found in permanent pathology, SLNB seemed "over-treatment". Under this concept, in our pre-OP DCIS patients, $32.6 \%$ were adequate-treated with SLNB, $58.4 \%$ over-treated, and $1.5 \%$ under-treated. Another $7.6 \%$ patients with post-OP pure DCIS did not receive surgical axillary biopsy, which should view as adequateobservation. If only "invasive component" present in final pathology viewed as an indication for SLNB, then up to $60 \%$ of patients diagnosed with pre-OP DCIS received inadequate axillary management (Table 2). This highlights the "unmet medical need" in modern era of personalized breast cancer care, which over-treated the low risk and/or low disease burden axilla. 
In post-OP pure DICS patients, $0.8 \%$ ALN metastasis rate is not justified for surgical ALN biopsy. In pre-OP invasive cancer patients, ALN metastasis rate could up to $39 \%$ and surgical lymph node biopsy with either SLN and/or ALND is indicated (Table 2). The group of patients, who diagnosed initially as DCIS, had a substantial risk of upgrade to invasive cancer (34.2\% in current study) and $7.6 \%$ overall lymph node metastasis cases detected. In patients of DCIS-IC, the ALN metastasis rate up to $20.6 \%$. One could speculate that patients with biopsy proven pre-OP DCIS present a category of "low" (pure DCIS) to "intermediate" (DCIS-IC) risk of ALN metastasis, which possessed some controversy whether routine lymph node surgery should be performed and it remained unsolved issue even in current modern breast imaging era.

According to NCCN and other practice guideline, patients with pre-OP biopsy diagnosed DCIS, selected for BCS and adjuvant radiotherapy, do not routinely indicate for SLNB[30]. As most patients (77\% in current study) with pre-OP biopsy diagnosed DCIS received BCS would remain pure DCIS in final pathology report (Fig. 1, Table 2). Furthermore, according to ACOSZ0011 trial, even 1-2 positive sentinel lymph node present, patients could omit ALND if breast cancer with tumor size T1-T2, received BCS and whole breast radiotherapy would be performed[22]. These reasons supported that SLNB is not routinely needed in preOP biopsy diagnosed DCIS patients indicated for BCS (Table 2).

However, some patients with pre-OP biopsy diagnosed DCIS and indicated for BCS would have post-op upgrade to DCIS-IC and with more than 2 lymph nodes metastases. In current study, we observed 7 pre-OP DCIS patients who received BCS presented with ALN metastases in final pathology check-up. Five of them were N1, 1 was N2, and the other 1 was N3 status (Fig. 1). This accounts that about $2.7 \%(7 / 252)$ of preOP DCIS and indicated BCS patients would have ALN metastasis. Although only few patients would have $>2$ occult metastatic lymph nodes, routinely abandon SLNB is also worrisome.

Controversy did persist about the role of SLNB in patients with pre-OP biopsy diagnosed DCIS and indicated for mastectomy. Parallel with increasing early detection of breast cancer, DCIS rate increased up to $14 \%-20 \%$, and around half ( $68 \%$ in current study) of them would receive mastectomy. This group of patients constituted about $5 \%$ of annual breast cancer cases, which constituted a non-ignorable minority[6]. Current practice guideline had suggested SLNB to be performed in pre-OP biopsy diagnosed DCIS and received mastectomy as secondary lymph node staging surgery seems unreliable when post-OP pathology upgrade to invasive breast cancer[31, 32].

In our 430 patients with pre-OP biopsy diagnosed DCIS received mastectomy, 59.3\% remained DCIS-postOP, and in these patients SLNB seemed unnecessary or "over-treatment". About $40.7 \%(175 / 430)$ of patients with pre-OP DCIS received mastectomy and upgrade to DCIS-IC post-OP. Among these 175 patients, 171 (98\%) received SLNB, and 40 (23.5\%) was found to have LN metastasis. This accounts for 9.8\% (42/430) of pre-OP biopsy diagnosed DCIS and indicated mastectomy patients would have ALN metastasis. This result is consisted with Price et al.'s study, which showed about $10 \%$ patients would have $\geqq 1 \mathrm{LN}$ metastasis when SLNB was performed in patients with pre-OP biopsy diagnosed DCIS and indicated for mastectomy[6]. 
The reason that mastectomy patients tend to receive SLNB is that the upgrade rate is higher in patients received total mastectomy than partial mastectomy (BCS) $(40.7 \%$ versus $23 \%, P<0.01$, Table 2$)$. This is related to more multicentric breast cancer, more larger tumor size of patients would receive mastectomy than BCS, and larger tumor or multicentric lesions would possess higher risk lead to upgrade to DCIS-IC. Among the predictors of ALN metastasis for patients received SLNB, patients with post-OP invasive component was the highest risk of lymph node metastasis (Table 5).

In current study, we tried to evaluate the pre-operative MRI for pre-OP DCIS patients, and we had 386 patients with detail ALN evaluation and post-operative pathologic report were analyzed for concordance. The accuracy of MRI: sensitivity $53.8 \%$, specificity $77.8 \%$, PPV $14.9 \%$, NPV $95.9 \%$, accuracy $76.2 \%$. This high NPV rate is very useful, especial for patients selected for BCS patients. In patients with MRI estimated tumor size $\nabla 3 \mathrm{~cm}$ \& lymph node negative cases, the NPV of ALN metastasis is high (up to $96.4 \%)$, and all $(3.6 \%, 5 / 137)$ the FN cases were limited to $N 1$ (with 1-2 positive nodes only). Our data supported that pre-OP MRI evaluated node negative patients suitable for BCS patients could safety omitted SLNB if whole breast radiotherapy is to be performed. In mastectomy patients, this high NPV could be used as shared decision making, however, could not guarantee metastatic free lymph node to omit SLNB (Table 2).

Our current study is limited in its retrospective study and limited cases, which could not answer whether subgroup of patients could omit axillary surgery in pre-OP DCIS patients indicated for mastectomy. Our current study, however, did collect of 682 pre-OP DCIS patients with detailed pre-op and post-OP lymph node pathologic report. Of special note that we had 386 patients with detailed pre-OP breast MRI evaluation and post-operative pathologic results, which enable us to evaluate the role of breast MRI in decision of lymph node surgery.

\section{Conclusion}

Individualized lymph node evaluation/biopsy of pre-OP DCIS patients is warranted due to inherent specimen biopsy error, which lead to substantial upgrade to invasive component found in final pathology, and therefore raise the concern of axillary lymph node metastasis. Patients indicated for mastectomy deserved a more tailored planning as $50 \%$ SLNB was not necessary, $40 \%$ for staging purpose, and around $10 \%$ therapeutic. Pre-OP diagnosed DCIS patients with MRI tumor size $<3 \mathrm{~cm}$ and node negative condition suitable for BCS could safety omitted SLNB if whole breast radiotherapy is to be performed.

\section{Abbreviations}

\section{ALN}

axillary lymph node

DCIS

ductal carcinoma in situ

MRI 
magnetic resonance imaging

NPV

negative predictive value

PPV

positive predictive value

SLNB

sentinel lymph node biopsy

ALND

axillary lymph node dissection

CNB

core needle biopsy

VACB

vacuum-assisted core biopsy

IC

invasive component

BCS

breast conserving surgery

\section{Declarations}

\section{Author Disclosures}

None of the authors have conflicts of interest or financial ties to disclose.

\section{Financial Disclosure}

Funding: This study was funded by the Ministry of Science and Technology of Taiwan, and the number of this funding was: MOST 108-2314-B-371-006-. This study was also sponsored by research funding provided by the Changhua Christian Hospital 106-CCH-IRP-014, 106-CCH-IRP-015, 107-CCH-HCR-035, and 108-CCH-IRP-122.

\section{Acknowledgement}

The authors would like thank Shu-Hsin Pai, Yi-Ru Ke, Chin-Mei Tai, Yun-Ting Chang, Jia-Dan Hsu, and Ying-Ru Lai for the assistance in this study.

\section{References}

\section{References:}

1. Heymans C, van Bastelaar J, Visschers RGJ, Vissers YLJ: Sentinel Node Procedure Obsolete in Lumpectomy for Ductal Carcinoma In Situ. Clin Breast Cancer 2017, 17:e87-e93. 
2. Caswell-Smith P, Wall M: Ductal carcinoma in situ: Is core needle biopsy ever enough? J Med Imaging Radiat Oncol 2017, 61:29-33.

3. Podoll MB, Reisenbichler ES, Roland L, Bruner A, Mizuguchi S, Sanders MAG: Feasibility of the Less Is More Approach in Treating Low-Risk Ductal Carcinoma In Situ Diagnosed on Core Needle Biopsy: Ten-Year Review of Ductal Carcinoma In Situ Upgraded to Invasion at Surgery. Arch Pathol Lab Med 2018, 142:1120-1126.

4. Munck F, Clausen EW, Balslev E, Kroman N, Tvedskov TF, Holm-Rasmussen EV: Multicentre study of the risk of invasive cancer and use of sentinel node biopsy in women with a preoperative diagnosis of ductal carcinoma in situ. Br J Surg 2020, 107:96-102.

5. Prendeville S, Ryan C, Feeley L, O'Connell F, Browne TJ, O'Sullivan MJ, Bennett MW: Sentinel lymph node biopsy is not warranted following a core needle biopsy diagnosis of ductal carcinoma in situ (DCIS) of the breast. Breast 2015, 24:197-200.

6. Price A, Schnabel F, Chun J, Kaplowitz E, Goodgal J, Guth A, Axelrod D, Shapiro R, Mema E, Moy L, et al: Sentinel lymph node positivity in patients undergoing mastectomies for ductal carcinoma in situ (DCIS). Breast J 2020, 26:931-936.

7. Goyal A, Douglas-Jones A, Monypenny I, Sweetland H, Stevens G, Mansel RE: Is there a role of sentinel lymph node biopsy in ductal carcinoma in situ?: analysis of 587 cases. Breast Cancer Res Treat 2006, 98:311-314.

8. Yen TW, Hunt KK, Ross MI, Mirza NQ, Babiera GV, Meric-Bernstam F, Singletary SE, Symmans WF, Giordano SH, Feig BW, et al: Predictors of invasive breast cancer in patients with an initial diagnosis of ductal carcinoma in situ: a guide to selective use of sentinel lymph node biopsy in management of ductal carcinoma in situ. J Am Coll Surg 2005, 200:516-526.

9. Shin YJ, Kim SM, Yun B, Jang M, Kim B, Lee SH: Predictors of Invasive Breast Cancer in Patients With Ductal Carcinoma In Situ in Ultrasound-Guided Core Needle Biopsy. J Ultrasound Med 2019, 38:481488.

10. Nakhlis F, Harrison BT, Giess CS, Lester SC, Hughes KS, Coopey SB, King TA: Evaluating the Rate of Upgrade to Invasive Breast Cancer and/or Ductal Carcinoma In Situ Following a Core Biopsy Diagnosis of Non-classic Lobular Carcinoma In Situ. Ann Surg Oncol 2019, 26:55-61.

11. Lamb LR, Lehman CD, Oseni TO, Bahl M: Ductal Carcinoma In Situ (DCIS) at Breast MRI: Predictors of Upgrade to Invasive Carcinoma. Acad Radiol 2019.

12. Lamb LR, Kim G, Oseni TO, Bahl M: Noncalcified Ductal Carcinoma In Situ (DCIS): Rate and Predictors of Upgrade to Invasive Carcinoma. Acad Radiol 2020.

13. Sim YT, Litherland J, Lindsay E, Hendry P, Brauer K, Dobson H, Cordiner C, Gagliardi T, Smart L: Upgrade of ductal carcinoma in situ on core biopsies to invasive disease at final surgery: a retrospective review across the Scottish Breast Screening Programme. Clin Radio/ 2015, 70:502-506.

14. Chin-Lenn L, Mack LA, Temple W, Cherniak W, Quinn RR, Ravani P, Lewin AM, Quan ML: Predictors of treatment with mastectomy, use of sentinel lymph node biopsy and upstaging to invasive cancer in 
patients diagnosed with breast ductal carcinoma in situ (DCIS) on core biopsy. Ann Surg Oncol 2014, 21:66-73.

15. Sorrentino L, Sartani A, Bossi D, Amadori R, Nebuloni M, Truffı M, Bonzini M, Riggio E, Foschi D, Corsi F: Sentinel node biopsy in ductal carcinoma in situ of the breast: Never justified? Breast J 2018, 24:325-333.

16. Watanabe Y, Anan K, Saimura M, Koga K, Fujino M, Mine M, Tamiya S, Nishihara K, Nakano T, Mitsuyama S: Upstaging to invasive ductal carcinoma after mastectomy for ductal carcinoma in situ: predictive factors and role of sentinel lymph node biopsy. Breast Cancer 2018, 25:663-670.

17. Allen A, Cauthen A, Dale P, Jean-Louis C, Lord A, Smith B: Evaluating the frequency of upgrade to malignancy following surgical excision of high-risk breast lesions and ductal carcinoma in situ identified by core needle biopsy. Breast J 2019, 25:103-106.

18. Verbelen $\mathrm{H}, \mathrm{Tj}$ alma W, Meirte J, Gebruers $\mathrm{N}$ : Long-term morbidity after a negative sentinel node in breast cancer patients. Eur J Cancer Care (Engl) 2019, 28:e13077.

19. Verbelen H, Gebruers N, Eeckhout FM, Verlinden K, Tjalma W: Shoulder and arm morbidity in sentinel node-negative breast cancer patients: a systematic review. Breast Cancer Res Treat 2014, 144:21-31.

20. De Groef A, Van Kampen M, Tieto E, Schonweger P, Christiaens MR, Neven P, Geraerts I, Gebruers N, Devoogdt N: Arm lymphoedema and upper limb impairments in sentinel node-negative breast cancer patients: A one year follow-up study. Breast 2016, 29:102-108.

21. Edge SB, Sheldon DG: Counterpoint: Sentinel Lymph Node Biopsy Is Not Indicated for Ductal Carcinoma In Situ. 2003, 1:207.

22. Giuliano AE, Ballman KV, McCall L, Beitsch PD, Brennan MB, Kelemen PR, Ollila DW, Hansen NM, Whitworth PW, Blumencranz PW, et al: Effect of Axillary Dissection vs No Axillary Dissection on 10Year Overall Survival Among Women With Invasive Breast Cancer and Sentinel Node Metastasis: The ACOSOG Z0011 (Alliance) Randomized Clinical Trial. JAMA 2017, 318:918-926.

23. van Roozendaal LM, Goorts B, Klinkert M, Keymeulen K, De Vries B, Strobbe LJA, Wauters CAP, van Riet YE, Degreef E, Rutgers EJT, et al: Sentinel lymph node biopsy can be omitted in DCIS patients treated with breast conserving therapy. Breast Cancer Res Treat 2016, 156:517-525.

24. Sakr R, Barranger E, Antoine M, Prugnolle H, Darai E, Uzan S: Ductal carcinoma in situ: value of sentinel lymph node biopsy. J Surg Oncol 2006, 94:426-430.

25. Bertozzi S, Cedolini C, Londero AP, Baita B, Giacomuzzi F, Capobianco D, Tortelli M, Uzzau A, Mariuzzi L, Risaliti A: Sentinel lymph node biopsy in patients affected by breast ductal carcinoma in situ with and without microinvasion: Retrospective observational study. Medicine (Baltimore) 2019, 98:e13831.

26. Hotton J, Salleron J, Rauch P, Buhler J, Pierret M, Baumard F, Leufflen L, Marchal F: Predictive factors of axillary positive sentinel lymph node biopsy in extended ductal carcinoma in situ treated by simple mastectomy at once. J Gynecol Obstet Hum Reprod 2020, 49:101641.

27. Magnoni F, Massari G, Santomauro G, Bagnardi V, Pagan E, Peruzzotti G, Galimberti V, Veronesi P, Sacchini VS: Sentinel lymph node biopsy in microinvasive ductal carcinoma in situ. Br J Surg 2019, 
106:375-383.

28. Holm-Rasmussen EV, Jensen MB, Balslev E, Kroman N, Tvedskov TF: Risk factors of sentinel and non-sentinel lymph node metastases in patients with ductal carcinoma in situ of the breast: $A$ nationwide study. Breast 2018, 42:128-132.

29. Lee CW, Wu HK, Lai HW, Wu WP, Chen ST, Chen DR, Chen CJ, Kuo SJ: Preoperative clinicopathologic factors and breast magnetic resonance imaging features can predict ductal carcinoma in situ with invasive components. Eur J Radiol 2016, 85:780-789.

30. Lyman GH, Somerfield MR, Bosserman LD, Perkins CL, Weaver DL, Giuliano AE: Sentinel Lymph Node Biopsy for Patients With Early-Stage Breast Cancer: American Society of Clinical Oncology Clinical Practice Guideline Update. J Clin Oncol 2017, 35:561-564.

31. Cody HS, Zee KJV: Point: Sentinel Lymph Node Biopsy Is Indicated for Patients With DCIS. 2003, 1:199.

32. Cardoso F, Kyriakides S, Ohno S, Penault-Llorca F, Poortmans P, Rubio IT, Zackrisson S, Senkus E, clinicalguidelines@esmo.org EGCEa: Early breast cancer: ESMO Clinical Practice Guidelines for diagnosis, treatment and follow-updagger. Ann Oncol 2019, 30:1194-1220.

\section{Figures}

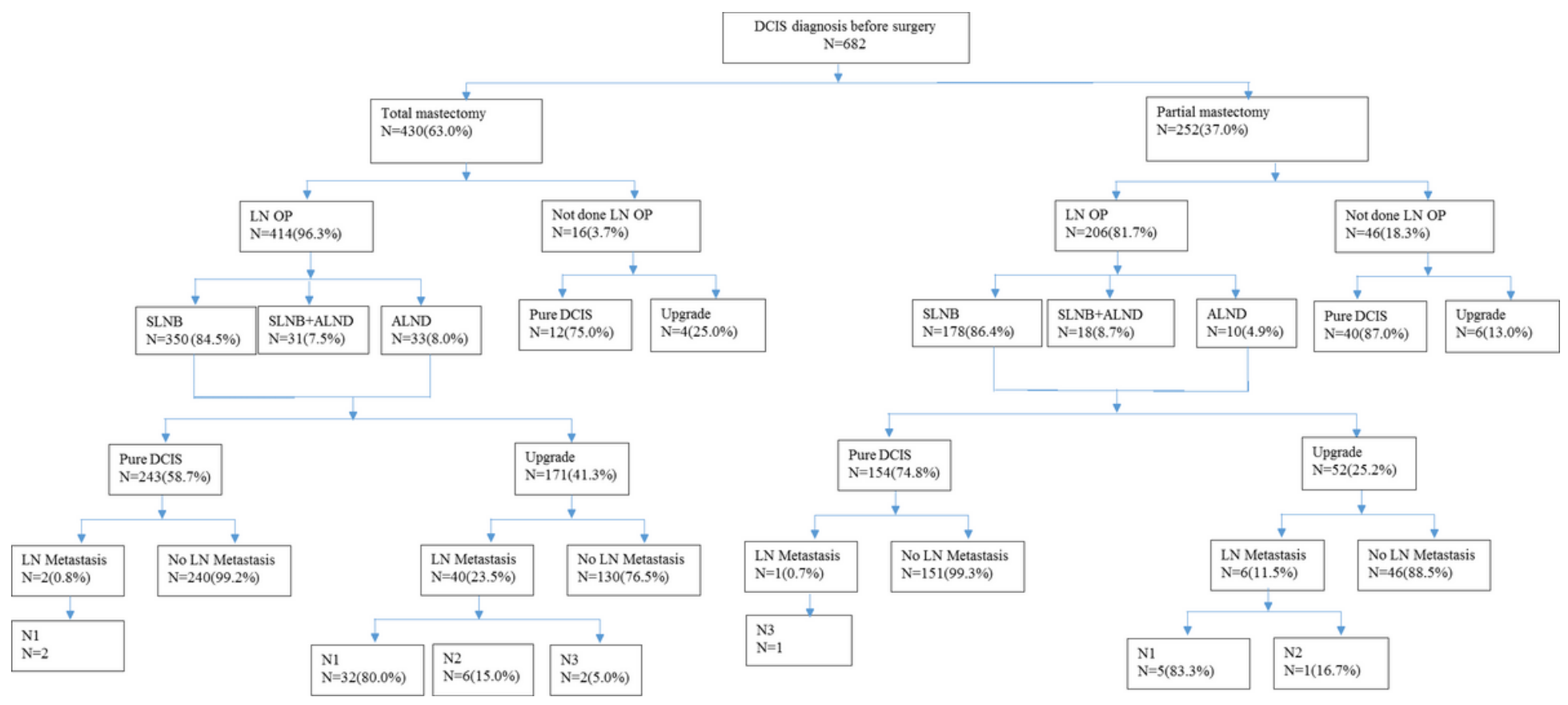

Figure 1

Flow chart of patients management of 682 pre-operative biopsy diagnosed ductal carcinoma in situ cases. The number of patients allocation to either breast conserving surgery (BCS) or mastectomy, whether received axillary lymph node surgery, pathology upgrade to invasive component or not, and postoperative lymph node status were described in details. 